\title{
Variability in Brain Activity as an Individual Difference Measure in Neuroscience?
}

\author{
Peter N. C. Mohr and Irene E. Nagel \\ Department of Education and Psychology, Freie Universität Berlin, 14195 Berlin, Germany, and Max Planck Institute for Human Development, Berlin, \\ 14195 Berlin, Germany \\ Review of Samanez-Larkin et al.
}

In neurobiological research it is common to identify effects for a certain population of individuals, thereby averaging across participants. Thus, the goal of a functional magnetic resonance imaging (fMRI) data analysis typically is to test whether an average task-induced change in the blood oxygen level-dependent (BOLD) signal is, over a group of participants, significantly different between two conditions. Importantly, though, accumulating evidence from behavioral studies suggests that there are individual differences in cognitive as well as emotional, social, and perceptual functioning. Person-to-person variation has been observed in several domains including personality traits, higher cognition, and attitudes. What is more, this person-to-person variation increases with age (MacDonald et al., 2006). Together, these findings point to the importance of considering individual differences in neurobiological research, particularly in the context of aging studies. In most fMRI studies, however, individual differences are neglected or simplified to group differences (e.g., between different age groups).

Only recently, there have been a few reports of studies with younger adults

Received March 26, 2010; revised April 15, 2010; accepted April 23, 2010. We thank Hauke R. Heekeren and Stuart W. MacDonald for fruitful discussions on the topic and comments on prior versions of the manuscript. Correspondence should be addressed to Peter N.C. Mohr, Department of Education and Psychology, Freie Universität Berlin, Habelschwerdter Allee 45, 14195 Berlin, Germany. E-mail: Peter.Mohr@fu-berlin.de. DOI:10.1523/JNEUROSCI.1560-10.2010

Copyright $\odot 2010$ the authors $\quad 0270-6474 / 10 / 307755-03 \$ 15.00 / 0$
(Hamann and Canli, 2004) and agecomparative settings that started taking individual differences into account. These studies aim to investigate the relationship between two variables (neurobiological and behavioral) that show substantial person-to-person variations. It should be noted, though, that in theses studies, analyses focused on differences in the average task-induced BOLD signal change of each subject. In these analyses variability around the BOLD signal is usually regarded as uninformative noise. However, in addition to changes in the average BOLD signal, also the variability around this signal could carry interesting information.

The most prominent metric to measure variability is the standard deviation (SD). It was recently applied to measure temporal variability around the average BOLD signal in an $\mathrm{MMRI}$ experiment. Garrett et al. (2010) compared the SD of the BOLD signal during fixation and its mean during this condition. They found that the age-predictive power of the whole-brain SD pattern was more than five times that of the mean, demonstrating the importance of variability measures especially in the context of aging. The SD is, however, only an appropriate measure of variability when the expected (mean) signal is constant, as for example during fixation. If the expected (mean) signal varies, as for example between different task conditions (e.g., fixation and stimulus presentation), the SD overestimates the variability around these different means (cf. Fig. 1).
It is based on the difference between single observations and the overall mean. Thus, it does not only take the variation around the different expected (mean) signals into account, but also the variation between the expected (mean) signals. This problem is circumvented by another measure of variability, namely the mean squared successive difference (MSSD). The MSSD is based on differences between successive observations. As the mean between successive observations constantly changes, the MSSD is able to adapt to changing expected (mean) signals. The MSSD can thus be regarded as an appropriate measure of temporal variability in experiments with different task conditions.

In their recent publication (2010), Samanez-Larkin and colleagues used temporal variability as an individual difference measure. More specifically, they investigated the relationship between individual differences in temporal variability of the BOLD signal and age-related suboptimal financial risk taking. The hypothesis was that older adults are impaired in risky decision making because age-related declines in dopamine levels lead to noisier signal processing in the striatum and thus to compromised value estimation during decision making. A representative group of participants was asked to conduct the Behavioral Investment Allocation Strategy (BIAS) task (Kuhnen and Knutson, 2005). In this task, participants choose repeatedly between two stocks and a bond, for which they received feedback regard- 
ing their outcomes afterward. In each block one stock was randomly assigned the "good" stock and the other one the "bad" stock, meaning that one stock dominated the other in the sense of first-order stochastic dominance, differing only in probabilities but not in possible outcome magnitudes. In the BIAS task, a rational, risk-neutral actor is assumed to constantly update her beliefs about the probability of each stock being optimal according to Bayes' rule. She will initially choose the bond and then after gaining enough evidence switch to the good stock. The BIAS task thus allows investigations of differences between actual behavior and a rational actor model. There are three types of deviation from the rational actor model that could occur in the BIAS task. Participants could either choose a stock when they should choose a bond (risk-seeking mistake) or choose a bond when a stock was the optimal choice (risk aversion mistakes). Finally, they could choose the bad stock although there was enough evidence in favor of the good stock (confusion mistakes).

In contrast to the popular view that older adults are more risk averse than younger adults, Samanez-Larkin and colleagues found that older subjects made more risk-seeking mistakes than younger subjects. They also made somewhat more confusion errors, but were equal in the amount of risk aversion errors. SamanezLarkin hypothesized that the effect of age on differences in the number of riskseeking mistakes can be explained by the amount of temporal variability in activation of the nucleus accumbens (NAcc), a brain region previously identified as a key brain structure in risk-seeking (Kuhnen and Knutson, 2005). A mediation analysis was conducted, which appropriately tests this sort of causal relation. The results of this analysis reveal that age differences cause differences in temporal variability in the NAcc which in turn cause differences in risk-seeking mistakes in the BIAS. Average task-induced NAcc activity, in contrast, did not predict risk-seeing mistakes, showing that variability in the BOLD signal might explain behavior better than the average task-induced BOLD signal.

Samanez-Larkin and colleagues suggest that one reason for higher temporal variability in the BOLD signal could be lower levels of dopamine expression in dopamine-sensitive brain regions such as the NAcc or the prefrontal cortex. This notion is based on the more general hypothesis that dopamine influences the signal-to-noise ratio of information pro-

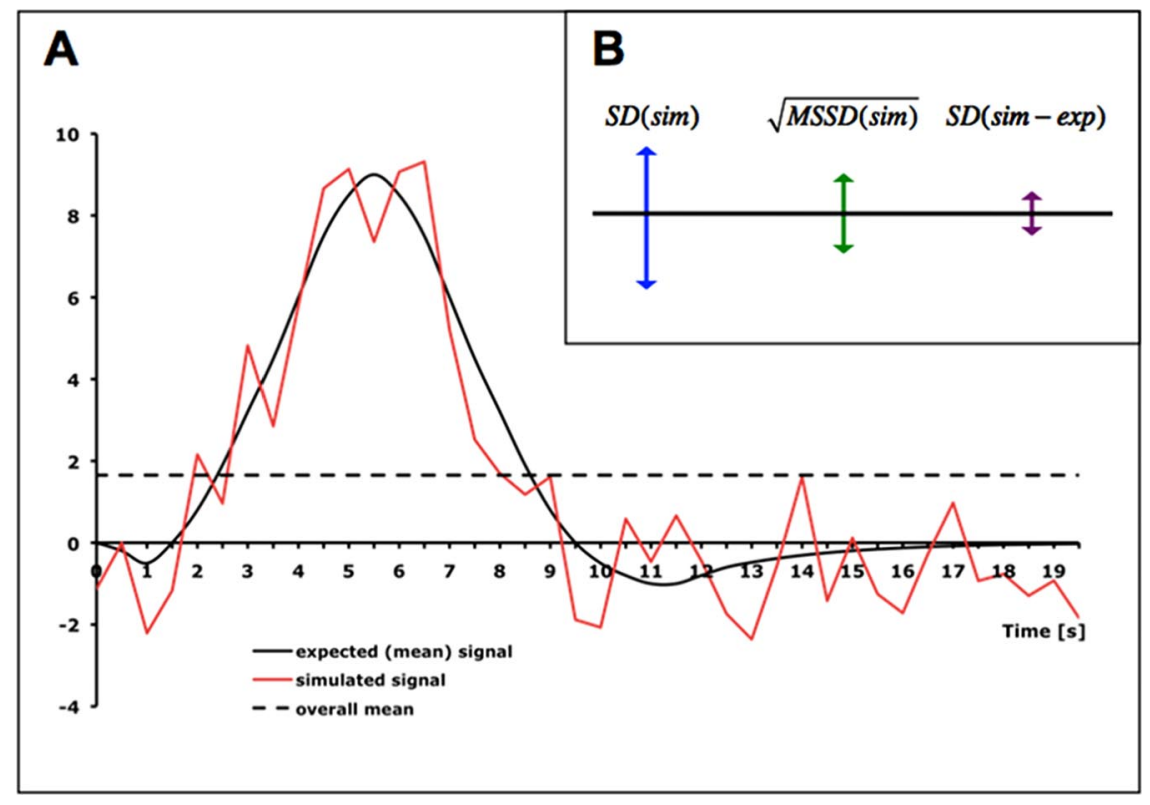

Figure 1. Comparison of variability measures for a simulated hemodynamic response. Variability can be measured by both the SD and the MSSD. While the SD often largely overestimates the true temporal variability, the MSSD is usually a good approximation of the true temporal variability. $\boldsymbol{A}$, The simulated signal of the hemodynamic response (here in red) varies around the expected (mean) signal (here in black). $\boldsymbol{B}$, The true temporal variability of the simulated signal in $\boldsymbol{A}$ can be described as the SD of the difference between the simulated signal and the expected (mean) signal [SD(sim - exp), size indicated by the purple arrow]. As the expected (mean) signal is unobservable, it is impossible to compute the true temporal variability of the simulated signal around it. The SD of the simulated signal [SD(sim), size indicated by the blue arrow] is based on the difference between the simulated signal and the overall mean (dashed line in $\boldsymbol{A}$ ). The SD of the simulated signal therefore largely overestimates the true temporal variability and is insensitive to changes in the expected (mean) signal. The MSSD of the simulated signal (size indicated by the green arrow), in contrast, is based on differences of successive observations of the simulated signal. It therefore takes changes in the expected (mean) signal into account and better approximates the true temporal variability of the signal.

cessing, thereby influencing the distinctiveness of representations in the brain and, ultimately, performance (Li et al., 2001). Evidence for this hypothesis comes from both neurocomputional and empirical investigations (MacDonald et al., 2009). Dopamine levels are increased or decreased relative to the normal population in various kinds of patient groups such as ADHD, Parkinson's disease, and schizophrenia. Importantly, dopamine levels also decrease during the course of normal aging, which may lead to a less efficient BOLD response in older adults. Finally, dopamine levels may also be altered by genetic differences; for example, by the catechol-methyl-transferase (COMT) gene. Depending on genetic predisposition, dopamine levels are slightly lower in some individuals, leading to decreased processing specificity in the prefrontal cortex (PFC), to greater variability of the BOLD signal, and to increases in behavioral variability. Due to the age-related declines of the dopamine system, these genotype-induced differences can be even more pronounced in older adults (Nagel et al., 2008), which might be one reason for the increased person-to-person variation in older adults.
Samanez-Larkin et al. (2010) thus found support for the hypothesis that older adults' impairment in risky decision making is due to a cascade of events where age-related declines in dopamine levels lead to noisy signal processing in the striatum, which in turn leads to compromised value estimation during decision making and thus ultimately to decreased performance in decision tasks. Importantly, with this study Samanez-Larkin and colleagues demonstrate the potential of individual difference analyses in neuroscience to explain interesting behavioral phenomena. What is more, they introduce a new measure of individual differences, namely temporal variability in the BOLD signal. As described above, the neurotransmitter dopamine is assumed to affect the temporal variability of the BOLD signal. Measures of temporal variability are therefore of particular interest when investigating individual differences in behaviors that are likely influenced by the dopamine system. Reward-based learning as well as value-based decision making rely heavily on dopaminergic target areas like the striatum and the ventromedial prefrontal cortex (VMPFC) (for review, see Mohr et al., 2010). Similarly, 
executive functions and working memory recruit prefrontal brain areas like the dorsolateral prefrontal cortex (DLPFC) and the dorsomedial prefrontal cortex (DMPFC) influenced by dopamine metabolism.

Individual differences in the temporal variability of the BOLD signal (as measured by the MSSD) might close the gap between observed individual differences in dopamine levels on the one hand and behavior on the other hand. Using such measures could shed light on one possible mechanism describing how dopamine level affects behavior, namely through its effect on temporal BOLD variability. To determine the exact mechanism, multimodal studies combining, for example, positron emission tomography (PET) with MRI, should examine the relation- ship between dopamine receptor binding and temporal BOLD variability to test directly whether increased temporal variability in the BOLD signal is caused by declines in the dopamine system.

\section{References}

Garrett DD, Kovacevic N, McIntosh AR, Grady CL (2010) Blood oxygen level-dependent signal variability is more than just noise. J Neurosci 30:4914-4921.

Hamann S, Canli T (2004) Individual differences in emotion processing. Curr Opin Neurobiol 14:233-238.

Kuhnen CM, Knutson B (2005) The neural basis of financial risk taking. Neuron 47:763-770.

Li SC, Lindenberger U, Sikstrom S (2001) Aging cognition: from neuromodulation to representation. Trends Cogn Sci 5:479-486.

MacDonald SW, Nyberg L, Backman L (2006) Intra-individual variability in behavior: links to brain structure, neurotransmission and neuronal activity. Trends Neurosci 29:474480.

MacDonald SW, Li SC, Backman L (2009) Neural underpinnings of within-person variability in cognitive functioning. Psychol Aging 24:792-808.

Mohr PN, Li SC, Heekeren HR (2010) Neuroeconomics and aging: neuromodulation of economic decision making in old age. Neurosci Biobehav Rev 34:678-688.

Nagel IE, Chicherio C, Li SC, von Oertzen T, Sander T, Villringer A, Heekeren HR, Bäckman L, Lindenberger U (2008) Human aging magnifies genetic effects on executive functioning and working memory. Front Hum Neurosci 2:1.

Samanez-Larkin GR, Kuhnen CM, Yoo DJ, Knutson B (2010) Variability in nucleus accumbens activity mediates age-related suboptimal financial risk taking. J Neurosci 30:14261434. 